\title{
How the Ocean Personality Model Affects the Perception of Crowds
}

\author{
Funda Durupınar = Bilkent University \\ Nuria Pelechano - Universitat Politècnica de Catalunya
}

Jan M. Allbeck - George Mason University

Uğur Güdükbay - Bilkent University

Norman I. Badler - University of Pennsylvania

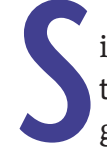
imulating the behavior of animated virtual crowds is a challenge for the computer graphics community. To achieve realistic behavior in virtual crowds requires extensive study of the semantics underlying real crowds' motion. Psychologists study human nature to identify salient behavior characteristics. There has been extensive research on incorporating psychological models into the simulation of autonomous agents. Here, however, we're not interested in a person's personality, per se, but in incorporating a personality model into large groups of people. By changing the parameters, we examine how subgroups of people with different personality traits interact and, accordingly, how global crowd behavior is influenced. The user decides the percentage and distribution of the personality traits.

Personality is a pattern of a person's behavioral, temperamental, emotional, and mental traits. Considerable controversy exists in personality research about how many personality traits there are. However, one popular model is the Five Factor, or Ocean (openness, conscientiousness, extroversion, agreeableness, and neu- roticism) model. ${ }^{1}$ These five factors are orthogonal dimensions of the personality space. Openness describes the imaginative and creative aspect of human character. Conscientiousness determines to what level a person is organized and careful. Extroversion relates to how outgoing and sociable a person is. Agreeableness is friendliness, generosity, and the tendency to get along with other people. Finally, neuroticism refers to emotional instability and the tendency to experience negative emotions. Each factor is bipolar and has several traits, which essentially are adjectives used to describe people. ${ }^{2}$

We've mapped these trait terms to the set of behaviors in the HiDAC (High-Density Autonomous Crowds) crowd simulation system. ${ }^{3}$ HiDAC models individual differences by assigning each person different psychological and physiological traits. Users normally set these parameters to model a crowd's nonuniformity and diversity. Our approach frees users of the tedious task of low-level parameter tuning and combines all these behaviors in distinct personality factors. To verify our mapping's plausibility, we evaluated users' perception of the personality traits in generated animations.

\section{The System}

By combining a standard personality model with a high-density crowd simulation, our approach creates plausible variations in the crowd and enables novice users to dictate these variations. ${ }^{4}$ 
Table 1. Low-level parameters versus trait-descriptive adjectives.

\begin{tabular}{|c|c|c|}
\hline Agent behavior & Personality factor & Ocean factor* \\
\hline Leadership & Assertive, social, unsocial, calm, fearful & $\mathrm{E}, \mathrm{N}$ \\
\hline Trained or untrained & Informed, ignorant & $\mathrm{O}$ \\
\hline Communication & Social, unsocial & $\mathrm{E}$ \\
\hline Panic & Oversensitive, fearful, calm, orderly, predictable & $\mathrm{N}, \mathrm{C}+$ \\
\hline Impatience & Rude, assertive, patient, stubborn, tolerant, orderly & $E+, C, A$ \\
\hline Pushing & Rude, kind, harsh, assertive, shy & $A, E$ \\
\hline Right preference & Cooperative, predictable, negative, contrary, changeable & $A, C$ \\
\hline Avoidance or personal space & Social, distant & $\mathrm{E}$ \\
\hline Waiting radius & Tolerant, patient, negative & A \\
\hline Waiting timer & Kind, patient, negative & A \\
\hline Exploring environment & Curious, narrow & $\mathrm{O}$ \\
\hline Walking speed & Energetic, lethargic, vigorless & $E$ \\
\hline Gesturing & Social, unsocial, shy, energetic, lethargic & $\mathrm{E}$ \\
\hline
\end{tabular}

\section{HIDAC}

HiDAC addresses the simulation of local behaviors and the global wayfinding of crowds in a dynamically changing environment. It directs autonomous agents' behavior by combining geometric and psychological rules. Psychological attributes include impatience, panic, and leadership behaviors, which are determined by traits such as locomotion, energy levels, and maximum speed. Agents have skills such as navigation in complex environments, communication, learning, and certain kinds of decision making. Agents also have perception so that they can react to obstacles, other agents, and dynamic changes in the environment.

To achieve realistic behavior, HiDAC handles collisions through avoidance and response forces. Over long distances, the system applies collision avoidance so that agents can steer around obstacles. Over shorter distances, it applies collision response to prevent agents from overlapping with each other and the environment.

Besides the usual crowd behavior, agents might display pushing behavior or show that they can wait for other agents to pass first, depending on their politeness and patience. Pushing behavior arises from varying each agent's personal-space threshold. Impatient agents don't respect others' personal space and appear to push their way through the crowd. Relaxed agents temporarily stop when another agent moves into their path; impatient agents don't respond to this feedback and tend to push.

\section{Integrating the Ocean Model into HiDAC}

A crowd consists of subgroups with different personalities. Variations in the subgroups' characteristics influence emergent crowd behavior. The user can add any number of groups with shared per- sonality traits and can edit these characteristics throughout an animation.

To model an agent's personality $\pi$, we use a fivedimensional vector, in which a personality factor $\Psi_{i}$ represents each dimension. To model the factors' distribution in a group of people, we use a Gaussian distribution function $N$ with mean $\mu_{i}$ and standard deviation $\sigma_{i}$ :

$\pi=\left\langle\Psi_{\mathrm{O}}, \Psi_{\mathrm{C}}, \Psi_{\mathrm{E}}, \Psi_{\mathrm{A}}, \Psi_{\mathrm{N}}>\right.$,

$\Psi_{i}=N\left(\mu_{i}, \sigma_{i}^{2}\right)$, for $i \in\{\mathrm{O}, \mathrm{C}, \mathrm{E}, \mathrm{A}, \mathrm{N}\}$,

where $\mu \in[0,1], \sigma \in[-0.1,0.1]$.

A person's overall behavior $\beta$ is a combination of different behaviors. Each behavior is a function of personality:

$\beta=\left(\beta_{1}, \beta_{2}, \ldots, \beta_{n}\right)$

$\beta_{j}=f(\pi)$, for $j=1, \ldots, n$.

Because each factor is bipolar, $\Psi$ can have positive and negative values. For instance, a value of 1 for extroversion means that the person is highly extroverted, whereas -1 means that the person is highly introverted.

\section{Personality-to-Behavior Mapping}

We map agents' personality factors (adjectives) onto low-level parameters and onto the builtin behaviors in the HiDAC model (see Table 1). A positive factor takes values in the range [0.5, 1]; a negative factor takes values in the range $[0$, 0.5). A factor with no sign indicates that both poles apply to that behavior. For instance, E+ for 
a behavior means that only extroversion is related to that behavior; introversion isn't applicable. As Table 1 shows, more than one personality dimension can define a behavior. The more adjectives of a certain factor that are defined for a behavior, the stronger that factor's impact on that behavior. We assign a weight to the factor's impact on a specific behavior. For instance, $\omega_{\mathrm{EL}}$ is the weight of extroversion on leadership; it takes a value in the range $[0,1]$. The sum of the weights for a specific type of behavior is 1 .

Now, we show how our approach maps a personality dimension to a specific type of behavior. We define the behavior parameters for an agent $i$ as follows.

Leadership. Leaders tend to have more confidence in themselves. They remain calm during emergencies. Each agent has a leadership percentage determined by extroversion and stability. We compute leadership behavior as

$$
\beta_{i}^{\text {Leadership }}=\omega_{\mathrm{EL}} \Psi_{i}^{\mathrm{E}}+\omega_{\mathrm{NL}}\left(1-\Psi_{i}^{\mathrm{N}}\right),
$$

where

$$
\beta_{i}^{\text {Leadership }} \propto \mathrm{E}, \beta_{i}^{\text {Leadership }} \propto^{-1} \mathrm{~N} \text {, and } \beta_{i}^{\text {Leadership }} \in[0,1] .
$$

Trained. Trained agents have complete knowledge about the environment. Because being trained requires curiosity and because trained people are informed, we associate this parameter with openness.

Being trained is a Boolean parameter, so we use a probability function to represent it. As openness increases, the probability that the agent is trained increases:

$$
\begin{aligned}
& P_{i}(\text { Trained })=\Psi_{i}^{O} \\
& \beta_{i}^{\text {Trained }}= \begin{cases}1 & \text { if } P_{i}(\text { Trained }) \geq 0.5 \\
0 & \text { otherwise }\end{cases}
\end{aligned}
$$

where

$P_{i}$ (Trained) $\propto \mathrm{O}$ and $\beta_{i}^{\text {Trained }} \in\{0,1\}$.

Communication. This parameter determines whether agents communicate. Similar to being trained, communication depends on the probability of agent behavior. As extroversion increases, the probability that the agent communicates increases:

$$
\begin{aligned}
& P_{i}(\text { Communication })=\Psi_{i}^{\mathrm{E}} \\
& \beta_{i}^{\text {Communication }}= \begin{cases}1 & \text { if } P_{i}(\text { Communication }) \geq 0.5 \\
0 & \text { otherwise }\end{cases}
\end{aligned}
$$

where

$P_{i}($ Communication $) \propto$ E and $\beta_{i}^{\text {Communication }} \in\{0,1\}$.

Panic. In emergency situations, agents display panic behavior depending on their stability and conscientiousness. When they panic, their walking speed increases and they don't wait. We compute panic as

$$
\begin{aligned}
& \beta_{i}^{\text {Panic }}=\omega_{\mathrm{NP}} \Psi_{i}^{\mathrm{N}}+\omega_{\mathrm{CP}} f\left(\Psi_{i}^{\mathrm{C}}\right) \\
& f\left(\Psi_{i}^{\mathrm{C}}\right)= \begin{cases}-2 \Psi_{i}^{\mathrm{C}}+2 & \text { if } \Psi_{i}^{\mathrm{C}} \geq 0 \\
0 & \text { otherwise }\end{cases}
\end{aligned}
$$

where

$\beta_{i}^{\text {Panic }} \propto \mathrm{N}, \beta_{i}^{\text {Panic }} \propto^{-1} \mathrm{C}+$, and $\beta_{i}^{\text {Panic }} \in[0,1]$.

Impatience. We implement this parameter by modifying the route selection dynamically on the basis of environmental changes. This parameter depends on an agent's politeness and assertiveness. We compute impatience as

$$
\begin{aligned}
& \beta_{i}^{\mathrm{Impatience}}=\omega_{\mathrm{EI}} f\left(\Psi_{i}^{\mathrm{E}}\right)+\omega_{\mathrm{AI}}\left(1-\Psi_{i}^{\mathrm{A}}\right)+\omega_{\mathrm{CI}}\left(1-\Psi_{i}^{\mathrm{C}}\right) \\
& f\left(\Psi_{i}^{\mathrm{E}}\right)= \begin{cases}2 \Psi_{i}^{\mathrm{E}}-1 & \text { if } \Psi_{i}^{\mathrm{E}} \geq 0 \\
0 & \text { otherwise }\end{cases}
\end{aligned}
$$

where

$$
\begin{aligned}
& \beta_{i}^{\text {Impatience }} \propto \mathrm{E}+, \beta_{i}^{\text {Impatience }} \\
& {[0,1] .}
\end{aligned}
$$

Pushing. HiDAC can realistically simulate a person's respect for others. Agents can try to force their way through a crowd by pushing others, exhibit more respectful behavior when desired, make decisions about letting others walk first, and queue when necessary. Disagreeable agents tend to push others more because they're harsh and impolite. Similarly, extroverted agents display pushing behavior because they tend to be assertive. We compute pushing as

$$
\begin{aligned}
& P_{i}(\text { Pushing })=\omega_{\mathrm{EP}} \Psi_{i}^{\mathrm{E}}+\omega_{\mathrm{AP}}\left(1-\Psi_{i}^{\mathrm{A}}\right) \\
& \beta_{i}^{\text {Pushing }}=\left\{\begin{array}{ll}
1 & \text { if } P_{i}(\text { Pushing }) \geq 0.5 \\
0 & \text { otherwise }
\end{array},\right.
\end{aligned}
$$

where

$P_{i}$ (Pushing) $\propto \quad \mathrm{E}, \quad P_{i}$ (Pushing) $\propto^{-1} \mathrm{~A}, \quad$ and
$\beta_{i}^{\text {Pushing }} \in\{0,1\}$. 
Right preference. People prefer to move toward the right side of the obstacle that they're about to encounter. This behavior shows the person's level of conformity to the rules. The right-preference behavior is a probability function. If an agent is disagreeable or nonconscientious, that agent can make a right or left preference with equal probability. On the other hand, an agent that prefers the right side increases the probability proportionally to the agent's agreeableness and conscientiousness values, if these values are positive. We compute right preference as

$$
\begin{aligned}
& P(\text { Right })= \begin{cases}0.5 & \text { if } \Psi_{i}^{\mathrm{A}}<0 \text { or } \Psi_{i}^{\mathrm{C}}<0 \\
\omega_{\mathrm{AR}} \Psi_{i}^{\mathrm{A}}+\omega_{\mathrm{CR}} \Psi_{i}^{\mathrm{C}} & \text { otherwise }\end{cases} \\
& \beta_{i}^{\text {Right }}=\left\{\begin{array}{ll}
1 \text { if } P_{i}(\text { Right }) \geq 0.5 \\
0 \text { otherwise }
\end{array},\right.
\end{aligned}
$$

where

$P_{i}$ (Right) $\propto \mathrm{A}, \mathrm{C}$, and $\beta_{i}^{\text {Right }} \in\{0,1\}$.

Personal space. Personal space determines the territory in which a person feels comfortable. Agents try to preserve their personal space when they approach other agents and when other agents approach from behind. However, the values for these two situations aren't the same. According to research, the average personal space in Western cultures is 0.7 meters in front and 0.4 meters behind. ${ }^{5}$ The personal space of an agent $i$ with respect to an agent $j$ is

$$
\begin{aligned}
& \beta_{i, j}^{\text {Personalspace }}= \begin{cases}0.8 * f(i, j) & \text { if } \Psi_{i}^{\mathrm{E}} \in\left[0 \frac{1}{3}\right) \\
0.7 * f(i, j) & \text { if } \Psi_{i}^{\mathrm{E}} \in\left[\frac{1}{3} \frac{2}{3}\right] \\
0.5 * f(i, j) & \text { if } \Psi_{i}^{\mathrm{E}} \in\left[\frac{2}{3} 1\right]\end{cases} \\
& f(i, j)= \begin{cases}1 & \text { if } i \text { is behind } j \\
0.4 / 0.7 & \text { otherwise }\end{cases}
\end{aligned}
$$

where

$$
\beta_{i, j}^{\text {PersonalSpace }} \propto^{-1} \mathrm{E} \text { and } \beta_{i, j}^{\text {PersonalSpace }} \in\{0.5,0.7,0.8\} .
$$

Waiting radius. In an organized situation, people tend to wait for available space before moving. We call this space the waiting radius; it depends on a person's kindness and consideration-that is, the agreeableness dimension. We compute the waiting radius as

$$
\beta_{i}^{\text {WaitingRadius }}= \begin{cases}0.25 & \text { if } \Psi_{i}^{\mathrm{A}} \in\left[0 \frac{1}{3}\right] \\ 0.45 & \text { if } \Psi_{i}^{\mathrm{A}} \in\left[\frac{1}{3} \frac{2}{3}\right] \\ 0.65 & \text { if } \Psi_{i}^{\mathrm{A}} \in\left(\frac{2}{3} 1\right]\end{cases}
$$

where

$$
\beta_{i}^{\text {WaitingRadius }} \propto \mathrm{A} \text { and } \beta_{i}^{\text {WaitingRadius }} \in\{0.25,0.45,0.65\} .
$$

Waiting timer. If two people are heading in the same direction, each waits for the other to move first. The time they wait-that is, the duration during which they display patience toward each other-depends on their agreeableness. We compute the waiting time as

$$
\beta_{i}^{\text {WaitingTimer }}=\left\{\begin{array}{ll}
1 & \text { if } \Psi_{i}^{\mathrm{A}} \in\left[0 \frac{1}{3}\right) \\
5 & \text { if } \Psi_{i}^{\mathrm{A}} \in\left[\frac{1}{3} \frac{2}{3}\right] \\
50 & \text { if } \Psi_{i}^{\mathrm{A}} \in\left[\frac{2}{3} 1\right]
\end{array},\right.
$$

where

$$
\beta_{i}^{\text {WaitingTimer }} \propto \mathrm{A} \text { and } \beta_{i}^{\text {WaitingTimer }} \in\{1,5,50\} .
$$

Exploring the environment. HiDAC assigns people specific behaviors. The number of actions they complete depends on their curiosity. Open people will more likely explore different experiences and perform more actions. The openness factor determines the time during which a person explores the environment. The number of actions that a person completes increases by the degree of openness. We compute the exploring parameter as

$$
\begin{aligned}
& \beta_{i}^{\text {Exploring }}=10 \Psi_{i}^{O}, \\
& \text { where } \\
& \beta_{i}^{\text {Exploring }} \propto O \text { and } \beta_{i}^{\text {Exploring }} \in[0,10] .
\end{aligned}
$$

Walking speed. A person's energy level determines that person's maximum walking speed. Because extroverts tend to be more energetic and introverts more lethargic, the extroversion trait controls this parameter. We compute the walking speed as

$\beta_{i}^{\text {WalkingSpeed }}=\Psi_{i}^{\mathrm{E}}+1$

where

$\beta_{i}^{\text {WalkingSpeed }} \propto \mathrm{E}$ and $\beta_{i}^{\text {WalkingSpeed }} \in[1,2]$. 


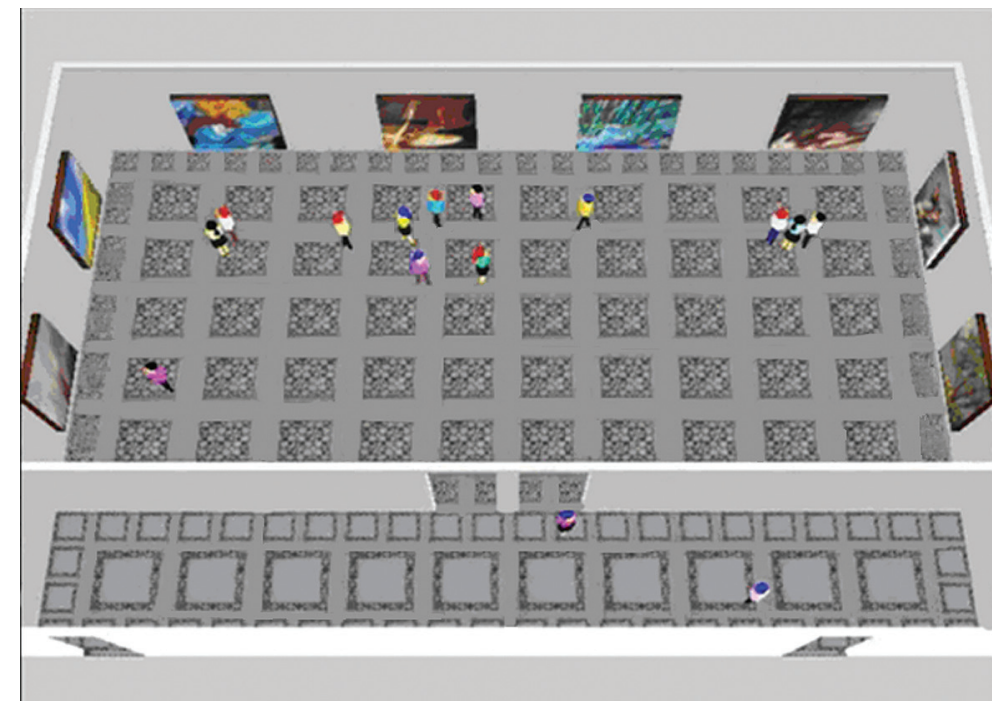

Figure 1. Openness tested in a museum. The most open people (red hair) stayed the longest. The least open people (blue hair) left the earliest.

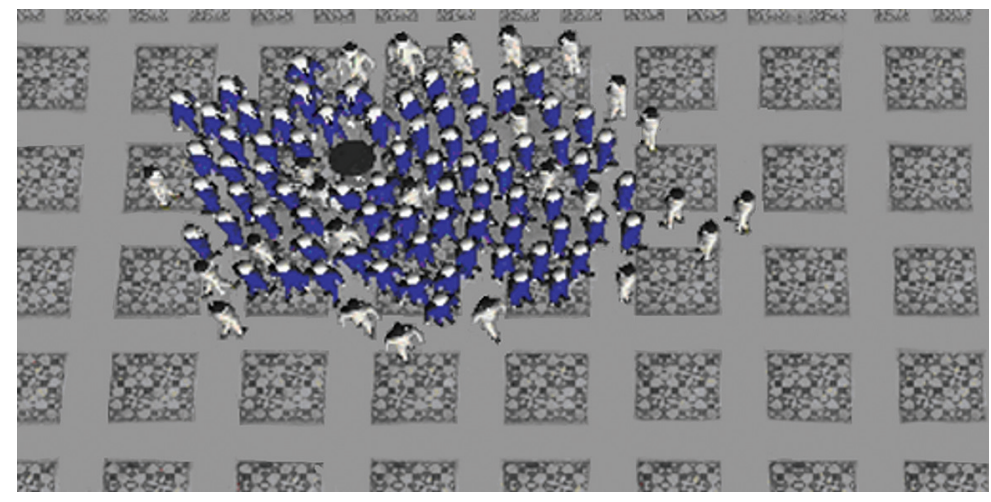

Figure 2. A ring formation example. Extroverts (blue suits) are inside and introverts are outside.

Gesturing. The amount of gesturing during a conversation indicates a person's sociability. Outgoing people use more gestures than shy people, which indicates extroversion. We compute the gesturing parameter as

$$
\beta_{i}^{\text {Gesturing }}=10 \Psi_{i}^{\mathrm{E}},
$$

where

$$
\beta_{i}^{\text {Gesturing }} \propto \mathrm{E} \text { and } \beta_{i}^{\text {Gesturing }} \in[0,10] .
$$

\section{Evaluation}

To evaluate whether users will correctly perceive our suggested mappings, we conducted user studies. We created several animations to study how modifying subgroups' personality parameters affects global crowd behavior.

\section{The Experiment's Design}

We created 15 videos presenting the emergent behaviors of people in scenarios in which the set- tings assigned in the Ocean model drive crowds' behavior. The scenarios ranged from evacuation drills to cocktail parties or museum galleries.

We performed the mapping from HiDAC parameters to Ocean factors by using trait-descriptive adjectives. To validate our system, we determined the correspondence between our mapping and the users' perception of these trait terms in the videos. Our studies involved 70 participants ( $21 \mathrm{fe}-$ males and 49 males, ages 18 to 30 ). We showed the videos to them on a projected display and asked them to complete a questionnaire containing 123 questions-about eight questions per video. After each video, participants had time to answer the related questions. The participants had no previous knowledge of the experiment.

Questions assessed how much a person agreed with statements such as, "I think the people in this video are kind" or "I think the people with green suits are calm." We asked questions that included the adjectives describing each Ocean factor instead of asking directly about the factors. We used descriptive questions because the general public, being unfamiliar with the Ocean model, might have difficulty answering questions such as, "Do the people exhibit openness?" Although the participants were proficient in English, to prevent any misconceptions, we attached dictionary definitions of the adjectives to the questionnaires. Participants chose answers on a scale from 0 to 10 , where $0=$ totally disagree, $5=$ neither agree nor disagree, and $10=$ totally agree. We omitted the antonyms from the list of adjectives, for conciseness. The remaining adjectives were assertive, calm, changeable, contrary, cooperative, curious, distant, energetic, harsh, ignorant, kind, orderly, patient, predictable, rude, shy, social, stubborn, and tolerant.

\section{Sample Scenarios}

In the scenarios, novel, emergent formations and different behavior timings occurred.

The museum scenario tested the impact of openness. A key factor determining openness is the belief in the importance of art. Figure 1 shows a screenshot from the sample animation. This scenario tested the adjectives curiosity and ignorance. There were three groups of people, with openness values of $0,0.5$, and 1 . We mapped the number of tasks that each agent must perform to openness, with each task requiring looking at a painting. The least open agents (with blue hair) left the museum first, followed by the agents with openness values of 0.5 (with black hair). The most open agents (with red hair) stayed the longest. We asked the participants how they perceived each group. 
Another video assessed how the participants perceived extroverts and introverts according to their distribution around a point of attraction. Figure 2 shows a screenshot in which the agents in blue suits are extroverted $(\mu=0.9$ and $\sigma=0.1$ ) and those in grey suits are introverted ( $\mu=0.1$ and $\sigma=0.1$ ). The ratio of introverts to extroverts in a society is 25 percent; ${ }^{6}$ we assigned the initial number of agents according to this ratio. At the animation's end, introverts were outside the ring structure around the object of attraction. Because extroverts are faster, they approached the attraction point in less time. In addition, when other agents blocked their way, they tended to push them to reach their goal. The figure also shows the difference between the personal spaces of introverts and extroverts. This animation tested the adjectives social, distant, assertive, energetic, and shy.

To test whether the participants could distinguish the personalities of people who create congestion, we showed them two videos of the same duration and asked them to compare the characteristics of the agents in each video. Each video consisted of two groups of people moving through each other. The first video showed people with high agreeableness and conscientiousness values $(\mu=0.9$ and $\sigma=0.1$ for both traits). The second video showed people with low agreeableness and conscientiousness values ( $\mu=0.1$ and $\sigma=0.1$ for both traits). In the first video, groups managed to cross each other, whereas in the second, congestion occurred after a fixed time period. Such behaviors emerged because people who are agreeable and conscientious are more patient; they don't push each other and are always predictable, because they prefer to move on the right side. Figure 3 shows how congestion occurred because of low conscientiousness and agreeableness. People were stuck at the center and refused to let other people move. They also were stubborn, negative, and uncooperative.

Figure 4 shows a screenshot from the animation demonstrating how neuroticism, nonconscientiousness, and disagreeableness affect panic. We simulated 13 agents. Five of them had neuroticism values of $\mu=0.9$ and $\sigma=0.1$, conscientiousness values of $\mu=0.1$ and $\sigma=0.1$, and agreeableness values of $\mu=0.1$ and $\sigma=0.1$. The other agents, which were psychologically stable, had neuroticism values of $\mu=0.1$ and $\sigma=0.1$, conscientiousness values of $\mu=0.9$ and $\sigma=0.1$, and agreeableness values of $\mu=0.9$ and $\sigma=0.1$. The agents in green suits are neurotic, less conscientious, and disagreeable. As the figure shows, they tend to panic more, push other agents, force their way through the crowd,
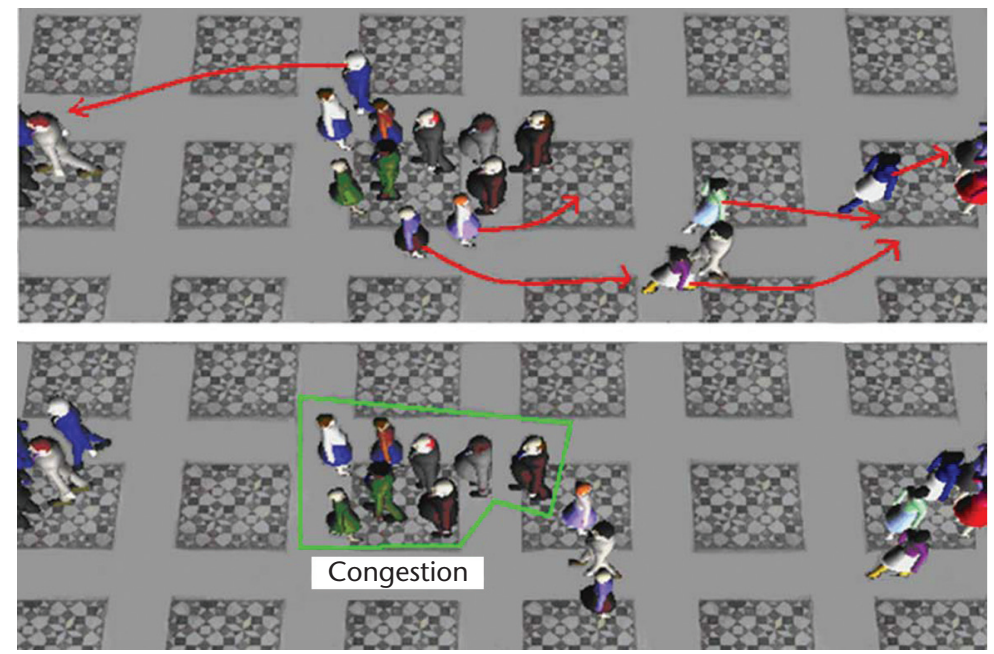

Figure 3. People with low conscientiousness and agreeableness cause congestion.
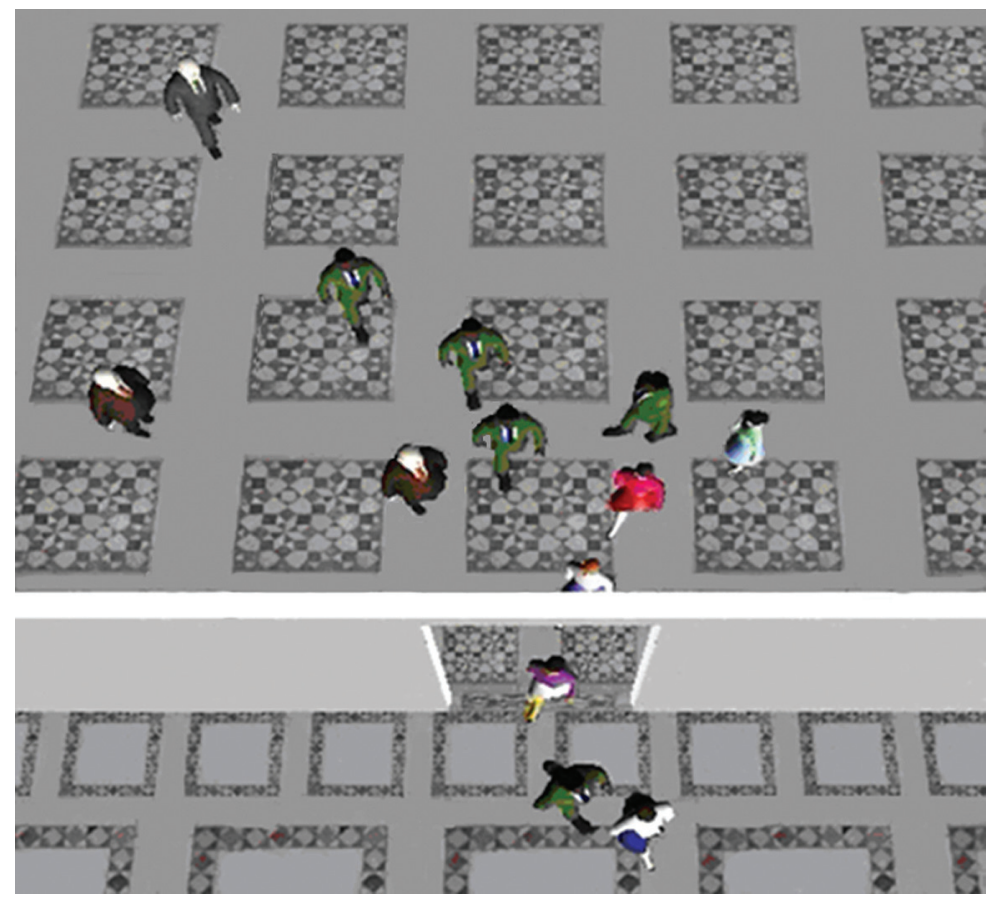

Figure 4. Neurotic, nonconscientious, and disagreeable agents (in green suits) display panic behavior.

and rush to the door. They aren't predictable, cooperative, patient, or calm; they're rude, changeable, negative, and stubborn.

\section{Analysis}

After collecting the participants' answers for all the videos, we organized the data for the adjectives. We classified each adjective by its question number, the simulation parameter, and the participants' answers to the corresponding question. We calculated the Pearson correlation ( $r$ ) between the simulation parameters and the average of the subjects' answers for each question. For instance, 


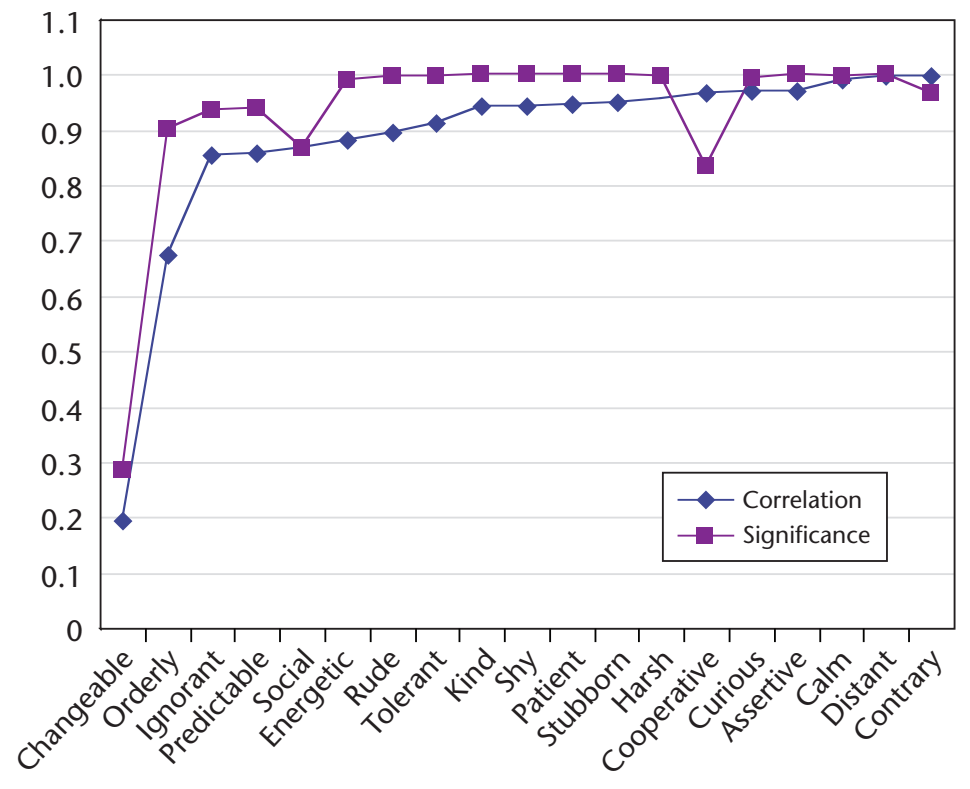

Figure 5. The correlation coefficients between the parameters and the subjects' answers for the descriptive adjectives (blue), and the significance values for the corresponding correlation coefficients (violet). Significance is low (<0.95) for changeable, orderly, ignorant, predictable, social, and cooperative.

eight questions included the adjective assertive, indicating a sample size of 8 . We calculated the correlation coefficient between the parameters and the means of the participants' answers between these 16 values, eight for each group.

Table 2. Correlation coefficients and significance values for the 12 adjectives.

\begin{tabular}{l|c|c}
\hline Adjective & Correlation & Significance \\
\hline Changeable & 0.199 & 0.288 \\
\hline Orderly & 0.674 & 0.903 \\
\hline Ignorant & 0.853 & 0.936 \\
\hline Predictable & 0.870 & 0.938 \\
\hline Social & 0.872 & 0.869 \\
\hline Energetic & 0.882 & 0.992 \\
\hline Rude & 0.897 & 0.997 \\
\hline Tolerant & 0.912 & 0.998 \\
\hline Kind & 0.943 & 1.000 \\
\hline Shy & 0.945 & 1.000 \\
\hline Patient & 0.948 & 1.000 \\
\hline Stubborn & 0.950 & 1.000 \\
\hline Harsh & 0.956 & 0.997 \\
\hline Cooperative & 0.967 & 0.834 \\
\hline Curious & 0.971 & 0.994 \\
\hline Assertive & 0.971 & 1.000 \\
\hline Calm & 0.988 & 0.999 \\
\hline Distant & 0.998 & 1.000 \\
\hline Contrary & 0.999 & 0.969 \\
\hline
\end{tabular}

We grouped the relevant adjectives for each Ocean factor to assess the perception of personality traits. This evaluation was similar to the evaluation of adjectives, this time considering the questions for all the adjectives that corresponded to an Ocean factor. For instance, because openness is related to curiosity and ignorance, we took into account the answers for curious and ignorant. Again, we averaged the subjects' answers for each question. Then, we computed the correlation with the parameters and the mean throughout all the questions involving curious and ignorant.

To estimate the probability of having obtained the correlation coefficients by chance, we computed the correlation coefficients' significance. The significance is $1-p$, where $p$ is the two-tailed probability, taking into account the sample size and the correlation value. Higher correlation and significance values suggest more accurate user perception.

\section{Results and Discussion}

Figure 5 shows the correlation coefficients and significance values for the adjectives; Table 2 shows the exact results. As the table shows, the significance is low $(<0.95)$ for changeable, orderly, ignorant, predictable, social, and cooperative. For changeable and orderly, this is because of low correlation values. For predictable, ignorant, social, and cooperative, the correlation coefficients are high, but their significance is low because of the small sample size.

From the participants' comments, we determined that changeable is especially confusing. To understand why, consider the setting in which two groups of agents crossed each other. The participants identified the nonconscientious agents as rude but perceived them as persistent in their rudeness. This perception caused the participants to mark lower values for the question about changeability. The same problem held for predictable agents. One participant's comments suggested that if a person is in a rush, that person can be predicted to push others. However, a predictable agent has a higher correlation despite these comments, even though predictable implies the opposite of changeable. This meaning might be because of the relatively low significance for predictable. Participants perceived nonconscientious agents that cause congestion as less predictable, which indicates that changing right-preference and rude behavior decreases the perceived predictability.

Orderly is another weakly correlated adjective. Analyzing the results for each video, we found that agents in the evacuation drill scenario were orderly although they displayed panic behavior. 
In these videos, even if the agents pushed each other and moved fast, some kind of order could be observed. This order was because of the crowd's smooth flow during evacuation. The crowd displayed collective synchrony, in which individuality was lost. Although people were impatient and rude, the overall crowd behavior appeared orderly.

We assigned the same goal to the entire crowd in the evacuation simulations because we aimed to observe disorganization locally. For instance, disorderly agents looked rushed; they pushed other agents and they didn't have solid preferences for selecting a direction when crossing another agent. However, they moved toward the same goal, which was to exit the building. The crowd would have appeared more disorderly if everyone ran in different directions and changed direction for no apparent reason.

Participants' answers suggest that they didn't recognize orderliness when the goal was the same for the whole crowd. On the other hand, in a scenario showing queuing behavior in front of a water dispenser, the participants could easily distinguish orderly versus disorderly people. Orderly agents waited at the end of the queue; disorderly agents rushed to the front. In this scenario, although the main goal was the same for all the agents (drink water), there were two distinguishable groups that acted differently.

Figure 6 and Table 3 show the correlation coefficients and their significance for the Ocean parameters. We computed these values by taking into account all the relevant adjectives for each Ocean factor. As the figure and table show, all the coefficients have high significance, with a probability of less than 0.5 percent of occurring by chance $(\mathrm{p}<0.005)$. The significance is high because we took into account all the adjectives describing a personality factor, thereby achieving a sufficiently large sample.

The correlation coefficient for conscientiousness is comparatively low, showing that the participants correctly perceived only approximately 44 percent of the traits $\left(r^{2} \approx 0.44\right)$. To understand why, consider the relevant adjectives: orderly, predictable, rude, and changeable. Low correlation values for orderly and changeable reduce the overall correlation. If we consider only rude and predictable, the correlation increases by 18.6 percent. The results suggest that people can observe the politeness aspect in short-term crowd behavior settings more easily than the organizational aspects. This observation also explains why the perception of agreeableness correlates highly with the actual parameters.

Figure 6 and Table 3 also show that the partici-

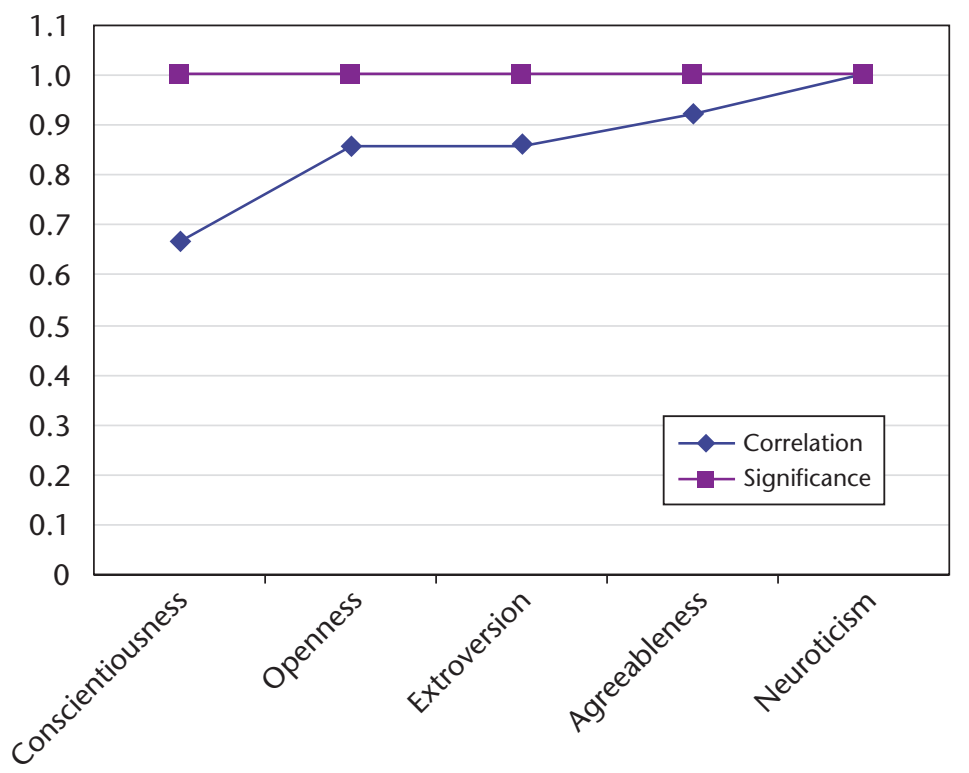

Figure 6. The correlation coefficients between actual parameters and subjects' answers for the Ocean factors (blue), and the two-tailed probability values for the corresponding correlation coefficients (violet). All the coefficients have high significance.

Table 3. The correlation coefficients and the significance values for the Ocean factors.

\begin{tabular}{|l|c|c|}
\hline Factor & Correlation & Significance \\
\hline Conscientiousness & 0.665 & 1.000 \\
\hline Openness & 0.859 & 0.999 \\
Extroversion & 0.860 & 1.000 \\
\hline Agreeableness & 0.922 & 1.000 \\
Neuroticism & 0.990 & 0.999 \\
\hline
\end{tabular}

pants perceived neuroticism the best. In this study, we've considered only neuroticism's calmness aspect, which is tested in emergency settings and building evacuation scenarios.

0 ur results are promising; they indicate a high correlation between our parameters and the participants' perception of them. The low correlation for some adjectives is due to the terms' ambiguity.

Unlike the low-level parameter tuning process in previous research (see the sidebar), we let our users select from higher-level concepts related to human psychology. Our approach frees users from understanding the underlying methodologies used in HiDAC. Our mapping also decreases the number of parameters to set, from 13 to 5 . Using a personality model let us move the user's focus to the agents' character instead of behavioral parameters, while providing us with a somewhat widely accepted structure for describing character. Certainly, you could create an interface that lets 
C rowd simulation research has evolved from creating reactive techniques to implementing crowds consisting of more complex agents. Reactive methods are limited; they don't present any knowledge representation, learning ability, reasoning, or individual differences in the agents. For instance, flocking systems are rule-based and specify an animation as a distributed global motion with a local tendency. ${ }^{1}$

On the other hand, systems with cognitive control involve reasoning and planning to accomplish long-term tasks, and they concentrate on achieving full autonomy. A notable step toward creating more intelligent agents was Xiaoyuan Tu and Demetri Terzopoulos's artificial-life simulation, which equipped artificial fishes with synthetic vision and perception of the environment, as well as behavior and learning centers. ${ }^{2}$ Soraia Musse and Daniel Thalmann proposed a crowd behavior model that implemented group interrelationships and introduced a multiresolution collision method specific to crowd modeling. ${ }^{3}$ Wei Shao and Terzopoulos introduced a complex pedestrian animation system that combined rule-based and cognitive models; it incorporated perceptual, behavioral, and cognitive control components. 4

Several studies have integrated emotion and personality models and roles into the simulation of autonomous agents, representing the individual differences through psychological states. Arjan Egges and his colleagues studied the simulation of the personality, emotions, and moods for conversational virtual humans. ${ }^{5}$ Taihua Li and colleagues proposed a framework that, like ours, uses the Ocean (openness, conscientiousness, extroversion, agreeableness, and neuroticism) model of personality to define and formulate a pedagogical agent in a social learning environment. ${ }^{6}$ However, these studies focused on individual agents, not crowds.
Only recently have researchers studied the perception of crowd variety. Christopher Peters and his colleagues evaluated pedestrians' perception. ${ }^{7}$ They determined how the orientation and context rules for characters in static scenes affect perceived plausibility. Rachel McDonnell and her colleagues analyzed the perceptual impact of the cloning of virtual characters for simulating large crowds. ${ }^{8}$

\section{References}

1. C. Reynolds, "Flocks, Herds, and Schools: A Distributed Behavior Model," Proc. Siggraph, ACM Press, 1987, pp. 25-34.

2. X. Tu and D. Terzopoulos, "Artificial Fishes: Physics, Locomotion, Perception, Behavior," Proc. Siggraph, ACM Press, 1994, pp. $43-50$.

3. S.R. Musse and D. Thalmann, "A Model of Human Crowd Behavior," Proc. Eurographics Workshop Computer Animation and Simulation, Springer, 1997, pp. 39-51.

4. W. Shao and D. Terzopoulos, "Autonomous Pedestrians," Graphical Models, vol. 69, nos. 5-6, 2007, pp. 246-274.

5. A. Egges, S. Kshirsagar, and N. Magnenat-Thalmann, "A Model for Personality and Emotion Simulation," Proc. Knowledge-Based Intelligence Information and Eng. Systems, LNCS 2773, Springer, 2003, pp. 453-461.

6. T. Li et al., "Modelling Personality, Emotion, and Mood for a Pedagogical Agent," Proc. 25th IASTED Int'l Multiconference: Artificial Intelligence and Applications (AIAP 07), ACTA Press, 2007, pp. 272-277.

7. C. Peters et al., "Crowds in Context: Evaluating the Perceptual Plausibility of Pedestrian Orientations," Proc. Eurographics, Short Papers, Eurographics Assoc., 2008, pp. 33-36.

8. R. McDonnell et al., "Clone Attack! Perception of Crowd Variety," ACM Trans. Graphics, vol. 27, no, 3, 2008, article 26. users create subgroups based on a set of adjectives instead of personality traits, but it would increase the number of parameters to set. Also, psychology and research on autonomous agents has linked personality models to other psychological, sociological, and cognitive models. Integrating a personality model into a crowd simulator will let us expand our simulator and explore how these other models affect crowd simulations.

We certainly could have used other psychological models. Autonomous-agent research has investigated emotion models. Future research might include adding emotion to the agents, but whereas personality is a behavior pattern (extended through time), emotions change according to the agent state and the situation. Emotions must evolve through the simulation and not be set by the animator. Certainly, personality af- fects emotional tendency and provides a foundation. Because personality is a behavior pattern, it might help a character's observers develop a sense of knowing that character. Thus, characters might become individuals instead of just another collection of anonymous computer characters.

\section{Acknowledgments}

The research described in this article was initiated while Funda Durupınar was visiting the University of Pennsylvania's Center for Human Modeling and Simulation. The Scientific and Technological Research Council of Turkey supported this research under International PhD Research Fellowship Programme 2214 and projects EEE-AG 104 E029 and 105E065. The Spanish Government partially funded this research under grant TIN2010-20590-C01-01. 


\section{References}

1. J.S. Wiggins, The Five-Factor Model of Personality: Theoretical Perspectives, Guilford Press, 1996.

2. L.R. Goldberg, "An Alternative 'Description of Personality': The Big-Five Factor Structure," J. Personality and Social Psychology, vol. 59, no. 6, 1992, pp. 1216-1229.

3. N. Pelechano, J.M. Allbeck, and N.I. Badler, "Controlling Individual Agents in High-Density Crowd Simulation," Proc. ACM Siggraph/Eurographics Symp. Computer Animation (SCA 07), ACM Press, 2007, pp. 99-108.

4. F. Durupinar et al., "Creating Crowd Variation with the Ocean Personality Model," Proc. 7th Int'l Joint Conf. Autonomous Agents and Multiagent Systems (AAMAS 08), Int'l Foundation for Autonomous Agents and Multiagent Systems, 2008, pp. 1217-1220.

5. E.T. Hall, The Hidden Dimension, Anchor Books, 1966.

6. K.C. McLean and M. Pasupathi, "Collaborative Narration of the Past and Extroversion," J. Research in Personality, vol. 40, no. 6, 2006, pp. 1219-1231.

Funda Durupınar received her PhD from the Department of Computer Engineering at Bilkent University. Her research interests include crowd simulation with heterogeneous behaviors that incorporate psychological aspects of agents. Durupinar has an MS in computer engineering from Bilkent University. Contact her at fundad@cs.bilkent.edu.tr.

Nuria Pelechano is an associate professor of Llenguatges $i$ Sistemes Informàtics at the Universitat Politècnica de Cata- lunya, where she's a member of the Moving and Event-Lab groups. Her research interests include simulation of crowds with heterogeneous behaviors, real-time 3D graphics, and human-avatar interaction in virtual environments. Pelechano has a PhD in computer and information science from the University of Pennsylvania. Contact her at npelechano@ lsi.upc.edu.

Jan M. Allbeck is an assistant professor of computer science at George Mason University. Her research interests are at the crossroads of animation, artificial intelligence, and psychology in the pursuit of simulating humans, including functional, heterogeneous crowds. Allbeck has a $\mathrm{PhD}$ in computer and information science from the University of Pennsylvania. Contact her at jallbeck@gmu.edu.

Ŭgur Güdükbay is an associate professor in Bilkent University's Department of Computer Engineering. His research interests include human modeling and animation, crowd simulation and visualization, and physically based modeling. Güdükbay has a $\mathrm{PhD}$ in computer engineering and information science from Bilkent University. He's a senior member of IEEE and the ACM. Contact him at gudukbay@ cs.bilkent.edu.tr.

Norman I. Badler is a professor of computer and information science at the University of Pennsylvania. He also directs the university's SIG Center for Computer Graphics and Center for Human Modeling and Simulation. His research interests center on computational connections between language and action. Badler has a PhD in computer science from the University of Toronto. Contact him at badler@seas. upenn.edu.

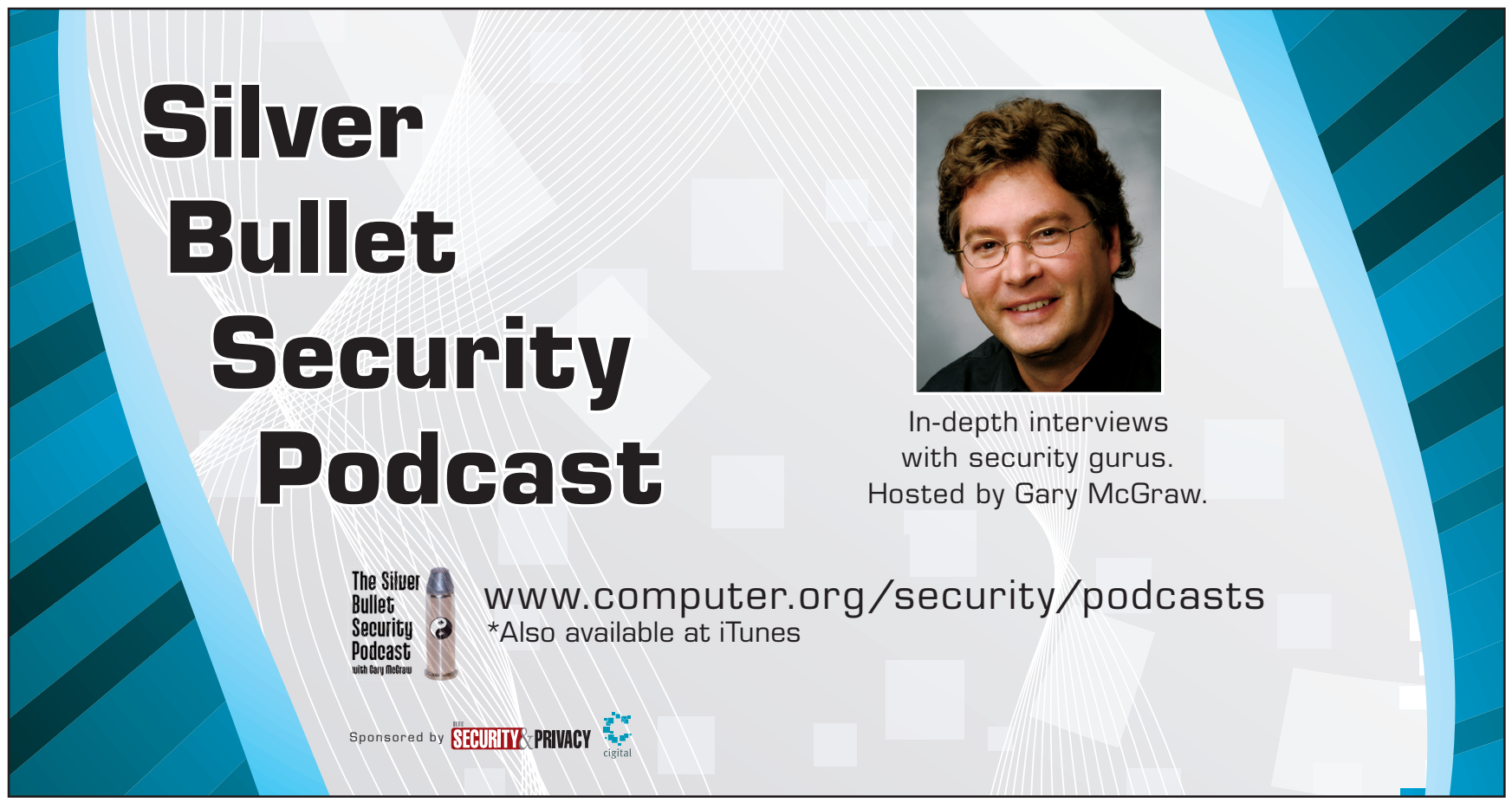

